\title{
Heavy flavour in ALICE
}

\author{
A. Dainese \\ (for the ALICE Collaboration) \\ INFN - Laboratori Nazionali di Legnaro, Legnaro (Padova), Italy
}

\begin{abstract}
The ALICE experiment, currently in the commissioning phase, will study nucleusnucleus and proton-proton collisions at the CERN Large Hadron Collider (LHC). We review the ALICE heavy-flavour physics program.
\end{abstract}

\section{Introduction}

ALICE $[1,2]$ is the dedicated heavy-ion experiment at the Large Hadron Collider (LHC). The main physics goal of the experiment is the study of strongly-interacting matter in the conditions of high energy density $\left(>10 \mathrm{GeV} / \mathrm{fm}^{3}\right)$ and high temperature $(\gtrsim 0.2 \mathrm{GeV})$, expected to be reached in central $\mathrm{Pb}-\mathrm{Pb}$ collisions at $\sqrt{s_{\mathrm{NN}}}=5.5 \mathrm{TeV}$. Under these conditions, according to lattice QCD calculations, quark confinement into colourless hadrons should be removed and a deconfined Quark-Gluon Plasma should be formed [1].

Heavy-flavour particles are regarded as effective probes of the system conditions. In particular:

- open charm and beauty hadrons would be sensitive to the energy density, through the mechanism of in-medium energy loss of heavy quarks;

- quarkonium states would be sensitive to the initial temperature of the system through their dissociation due to colour screening.

\section{Heavy-flavour phenomenology in nucleus-nucleus collisions at the LHC}

The expected $c \overline{\mathrm{c}}$ and $\mathrm{b} \overline{\mathrm{b}}$ production yields for pp collisions at $\sqrt{s}=14 \mathrm{TeV}$ are 0.16 and 0.0072 , respectively [2]. For the $5 \%$ most central $\mathrm{Pb}-\mathrm{Pb}$ collisions at $\sqrt{s_{\mathrm{NN}}}=5.5 \mathrm{TeV}$ the expected yields are 115 and 4.6, respectively. These numbers, assumed as the baseline for ALICE simulation studies, are obtained from pQCD calculations at NLO [3], including the nuclear modification of the parton distribution functions in the $\mathrm{Pb}$ nucleus. Note that the predicted yields have large uncertainties, of about a factor 2 , estimated by varying the values of the calculation parameters.

Experiments at the Relativistic Heavy Ion Collider (RHIC) have shown that the nuclear modification factor of particles $p_{\mathrm{t}}$ distributions, $R_{\mathrm{AA}}\left(p_{\mathrm{t}}, \eta\right)=\frac{1}{\left\langle N_{\mathrm{coll}}\right\rangle} \cdot \frac{\mathrm{d}^{2} N_{\mathrm{AA}} / \mathrm{d} p_{\mathrm{t}} \mathrm{d} \eta}{\mathrm{d}^{2} N_{\mathrm{pp}} / \mathrm{d} p_{\mathrm{t}} \mathrm{d} \eta}$, is a sensitive observable for the study of the interaction of the hard partons with the medium produced in nucleus-nucleus collisions. Heavy quark medium-induced quenching is one of the most captivating topics to be addressed in $\mathrm{Pb}-\mathrm{Pb}$ collisions at the LHC. Due to the QCD nature of parton energy loss, quarks are predicted to lose less energy than gluons (that have a higher colour charge) and, in addition, the 'dead-cone effect' is expected to reduce the energy loss of massive quarks with respect to light partons [4]. Therefore, one should observe a pattern of gradually decreasing $R_{\mathrm{AA}}$ suppression when going from the mostly gluon-originated 
light-flavour hadrons $\left(h^{ \pm}\right.$or $\left.\pi^{0}\right)$ to $\mathrm{D}$ and to $\mathrm{B}$ mesons: $R_{\mathrm{AA}}^{h} \lesssim R_{\mathrm{AA}}^{\mathrm{D}} \lesssim R_{\mathrm{AA}}^{\mathrm{B}}$. The measurement of $\mathrm{D}$ and $\mathrm{B}$ meson production cross sections will also serve as a baseline for the study of medium effects on quarkonia. Two of the most interesting items in the quarkonia sector at the LHC will be: (a) understanding the interplay between colour-screening-induced (and medium-temperature-dependent) suppression and statistical regeneration for $\mathrm{J} / \psi$ production in a medium containing on the order of $100 \mathrm{c} \overline{\mathrm{c}}$ pairs; (b) measuring for the first time medium effects on the bottomonia resonances, expected to be affected by colour-screening only if the temperature of the medium will be larger than about $0.4 \mathrm{GeV}$.

\section{$3 \quad$ Heavy-flavour detection in ALICE}

The ALICE experimental setup, described in detail in [1], allows the detection of open charm and beauty hadrons and of quarkonia in the high-multiplicity environment of central $\mathrm{Pb}-\mathrm{Pb}$ collisions at LHC energy, where a few thousand charged particles might be produced per unit of rapidity. The heavy-flavour capability of the ALICE detector is provided by:

- Tracking system; the Inner Tracking System (ITS), the Time Projection Chamber (TPC) and the Transition Radiation Detector (TRD), embedded in a magnetic field of $0.5 \mathrm{~T}$, allow track reconstruction in the pseudorapidity range $-0.9<\eta<0.9$ with a momentum resolution better than $2 \%$ for $p_{\mathrm{t}}<20 \mathrm{GeV} / c$ and a transverse impact parameter $^{\mathrm{a}}$ resolution better than $60 \mu \mathrm{m}$ for $p_{\mathrm{t}}>1 \mathrm{GeV} / c$ (the two innermost layers of the ITS, $r \approx 4$ and $7 \mathrm{~cm}$, are equipped with silicon pixel detectors).

- Particle identification system; charged hadrons are separated via $\mathrm{d} E / \mathrm{d} x$ in the TPC and via time-of-flight measurement in the Time Of Flight (TOF) detector; electrons are separated from charged hadrons in the dedicated Transition Radiation Detector (TRD), and in the TPC; muons are identified in the muon spectrometer covering the pseudo-rapidity range $-4<\eta<-2.5$.

Simulation studies [2] have shown that ALICE has good potential to carry out a rich heavy-flavour physics programme. The main analyses in preparation are:

- Open charm (section 4): fully reconstructed hadronic decays $\mathrm{D}^{0} \rightarrow \mathrm{K}^{-} \pi^{+}, \mathrm{D}^{+} \rightarrow$ $\mathrm{K}^{-} \pi^{+} \pi^{+}, \mathrm{D}_{\mathrm{s}}^{+} \rightarrow \mathrm{K}^{-} \mathrm{K}^{+} \pi^{+}$(under study), $\Lambda_{\mathrm{c}}^{+} \rightarrow \mathrm{pK}^{-} \pi^{+}$(under study) in $|\eta|<0.9$.

- Open beauty (section 4): inclusive single leptons $\mathrm{B} \rightarrow \mathrm{e}+X$ in $|\eta|<0.9$ and $\mathrm{B} \rightarrow \mu+X$ in $-4<\eta<-2.5$; inclusive displaced charmonia $\mathrm{B} \rightarrow \mathrm{J} / \psi\left(\rightarrow \mathrm{e}^{+} \mathrm{e}^{-}\right)+X$ (under study).

- Quarkonia (section 5): c $\overline{\mathrm{c}}\left(\mathrm{J} / \psi, \psi^{\prime}\right)$ and $\mathrm{b} \overline{\mathrm{b}}\left(\Upsilon, \Upsilon^{\prime}, \Upsilon^{\prime \prime}\right)$ states in the $\mathrm{e}^{+} \mathrm{e}^{-}(|\eta|<0.9)$ and $\mu^{+} \mu^{-}(-4<\eta<-2.5)$ channels.

For all simulation studies, a multiplicity $\mathrm{d} N_{\mathrm{ch}} / \mathrm{d} y=4000-6000$ was assumed for central $\mathrm{Pb}-\mathrm{Pb}$ collisions. In the following, we report the results corresponding to the expected statistics collected by ALICE per LHC year: $10^{7}$ central $\left(0-5 \% \sigma^{\text {inel }}\right) \mathrm{Pb}-\mathrm{Pb}$ events at $\mathcal{L}_{\mathrm{Pb}-\mathrm{Pb}}=10^{27} \mathrm{~cm}^{-2} \mathrm{~s}^{-1}$ and $10^{9} \mathrm{pp}$ events at $\mathcal{L}_{\mathrm{pp}}^{\mathrm{ALICE}}=5 \times 10^{30} \mathrm{~cm}^{-2} \mathrm{~s}^{-1}$, in the barrel detectors; the forward muon arm will collect about 40 times larger samples of muon-trigger events (i.e. $4 \times 10^{8}$ central $\mathrm{Pb}-\mathrm{Pb}$ events).

\footnotetext{
${ }^{a}$ The transverse impact parameter, $d_{0}$, is defined as the distance of closest approach of the track to the interaction vertex, in the plane transverse to the beam direction.
}

DIS 2008 

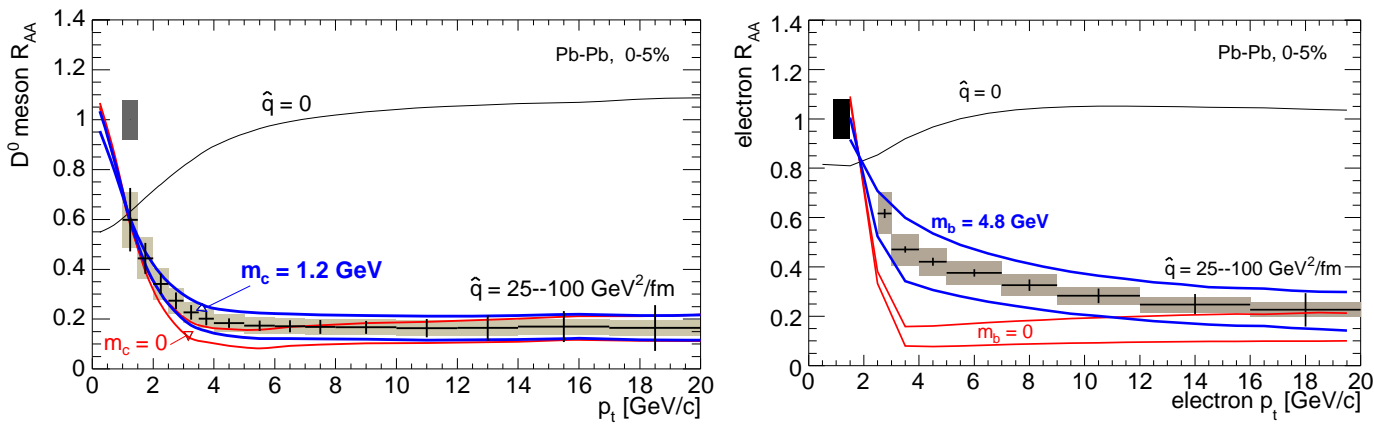

Figure 1: Nuclear modification factors for $\mathrm{D}^{0}$ mesons (left) and for B-decay electrons (right). Errors corresponding to the centre of the prediction bands for massive quarks are shown: bars $=$ statistical, shaded area $=$ systematic.

\section{Charm and beauty measurements}

Among the most promising channels for open charm detection are the $\mathrm{D}^{0} \rightarrow \mathrm{K}^{-} \pi^{+}(c \tau \approx$ $120 \mu \mathrm{m}$, branching ratio $\approx 3.8 \%)$ and $\mathrm{D}^{+} \rightarrow \mathrm{K}^{-} \pi^{+} \pi^{+}(c \tau \approx 300 \mu \mathrm{m}$, branching ratio $\approx$ $9.2 \%$ ) decays. The detection strategy to cope with the large combinatorial background from the underlying event is based on the selection of displaced-vertex topologies, i.e. separation from the primary vertex of the tracks from the secondary vertex and good alignment between the reconstructed D meson momentum and flight-line $[2,6]$. An invariant-mass analysis is used to extract the raw signal yield, to be then corrected for selection and reconstruction efficiency and for detector acceptance. The accessible $p_{\mathrm{t}}$ range for the $\mathrm{D}^{0}$ is $1-20 \mathrm{GeV} / c$ in $\mathrm{Pb}-\mathrm{Pb}$ and $0.5-20 \mathrm{GeV} / c$ in pp, with statistical errors better than $15-20 \%$ at high $p_{\mathrm{t}}$. Similar capability is expected for the $\mathrm{D}^{+}$. The systematic errors (acceptance and efficiency corrections, centrality selection for $\mathrm{Pb}-\mathrm{Pb}$ ) are expected to be smaller than $20 \%$.

The production of open beauty can be studied by detecting the semi-electronic decays of beauty hadrons, mostly $\mathrm{B}$ mesons. Such decays have a branching ratio of $\simeq 10 \%$. The main sources of background electrons are: decays of $\mathrm{D}$ mesons; $\pi^{0}$ Dalitz decays and decays of light vector mesons (e.g. $\rho$ and $\omega$ ); conversions of photons in the beam pipe or in the inner detector layer; pions misidentified as electrons. Given that electrons from beauty have average impact parameter $d_{0} \simeq 500 \mu \mathrm{m}$ and a hard $p_{\mathrm{t}}$ spectrum, it is possible to obtain a high-purity sample with a strategy that relies on: electron identification with a combined $\mathrm{d} E / \mathrm{d} x(\mathrm{TPC})$ and transition radiation (TRD) selection; impact parameter cut to reduce the charm-decay component and reject misidentified $\pi^{ \pm}$and $\mathrm{e}^{ \pm}$from Dalitz decays and $\gamma$ conversions. As an example, with $10^{7}$ central $\mathrm{Pb}-\mathrm{Pb}$ events, this strategy is expected to allow the measurement of electron-level $p_{\mathrm{t}}$-differential cross section in the range $2<p_{\mathrm{t}}<20 \mathrm{GeV} / c$ with statistical errors smaller than $15 \%$ at high $p_{\mathrm{t}}$. Similar performance figures are expected for pp collisions.

$\mathrm{B}$ production in $\mathrm{pp}$ and $\mathrm{Pb}-\mathrm{Pb}$ collisions can be measured also in the ALICE muon spectrometer $(-4<\eta<-2.5)$ analyzing the single-muon $p_{\mathrm{t}}$ distribution [2]. The main backgrounds to the 'beauty muon' signal are $\pi^{ \pm}, \mathrm{K}^{ \pm}$and charm decays. The cut $p_{\mathrm{t}}>$ $1.5 \mathrm{GeV} / c$ is applied to all reconstructed muons in order to increase the signal-to-background

DIS 2008 
ratio. Then, a fit technique allows to extract a $p_{\mathrm{t}}$ distribution of muons from $\mathrm{B}$ decays. Since only minimal cuts are applied, the statistical errors are expected to be smaller than $5 \%$ up to muon $p_{\mathrm{t}} \approx 30 \mathrm{GeV} / c$.

We investigated the possibility of using the described charm and beauty measurements to study the high- $p_{\mathrm{t}}$ suppression induced by parton energy loss. The sensitivity to $R_{\mathrm{AA}}^{\mathrm{D}}$ and $R_{\mathrm{AA}}^{\mathrm{e}}$ from B is presented in Fig. 1. Predictions [5] with and without the effect of the heavyquark mass, for a medium transport coefficient $\hat{q}$ (a measurement of the medium density) in the range $25-100 \mathrm{GeV}^{2} / \mathrm{fm}$, are also shown.

\section{Quarkonia capabilities}

ALICE can detect quarkonia in the dielectron channel at central rapidity $(|y| \lesssim 1)$ and in the dimuon channel at forward rapidity $(-4 \lesssim y \lesssim-2.5)$. In both channels the quarkonia acceptance extends down to zero transverse momentum, since the minimum $p_{\mathrm{t}}$ for e and $\mu$ identification is about $1 \mathrm{GeV} / c$. The high $p_{\mathrm{t}}$ reach is expected to be $10(20) \mathrm{GeV} / c$ for the $\mathrm{J} / \psi$ in $\mathrm{e}^{+} \mathrm{e}^{-}\left(\mu^{+} \mu^{-}\right)$, for a $\mathrm{Pb}-\mathrm{Pb}$ run of one month at nominal luminosity. In the bottomonium sector, the mass resolution of about $90 \mathrm{MeV}$ at $M_{\ell^{+} \ell^{-}} \sim 10 \mathrm{GeV}$, for both dielectrons and dimuons, should allow the separation of the $\Upsilon$ and $\Upsilon^{\prime}$ states, and thus the measurement of the $\Upsilon^{\prime} / \Upsilon$ ratio, which is expected to be sensitive to the initial temperature of the medium.

Simulation studies are in progress to prepare a measurement of the fraction of $\mathrm{J} / \psi$ that feed-down from B decays. Such measurement can be performed by studying the separation of the dilepton pairs in the $\mathrm{J} / \psi$ invariant mass region from the main interaction vertex. The analysis is also expected to provide a measurement of the beauty $p_{\mathrm{t}}$-differential cross section.

\section{Summary}

Heavy quarks, abundantly produced at LHC energies, will allow to address several issues at the heart of in heavy-ion physics. They provide tools to probe the density (via parton energy loss and its predicted mass dependence) and the temperature (via the dissociation patterns of quarkonia) of the high-density $\mathrm{QCD}$ medium formed in $\mathrm{Pb}-\mathrm{Pb}$ collisions. The

excellent tracking, vertexing and particle identification performance of ALICE will allow to fully explore this rich phenomenology.

\section{References}

[1] ALICE Collaboration, Physics Performance Report Vol. I, CERN/LHCC 2003-049 and J. Phys. G30 1517 (2003).

[2] ALICE Collaboration, Physics Performance Report Vol. II, CERN/LHCC 2005-030 and J. Phys. G32 1295 (2006).

[3] M.L. Mangano, P. Nason and G. Ridolfi, Nucl. Phys. B373 295 (1992).

[4] Yu.L. Dokshitzer and D.E. Kharzeev, Phys. Lett. B519 199 (2001).

[5] N. Armesto, et al., Phys. Rev. D71 054027 (2005).

[6] E. Bruna, Int. J. Mod. Phys. E16 2097 (2007). 